\title{
Asymptotics of Coupled Reaction-Diffusion Fronts with Multiple Static and Diffusing Reactants: Uranium Oxidation in Water Vapor.
}

DOI:

10.1137/19M1309791

\section{Document Version}

Accepted author manuscript

Link to publication record in Manchester Research Explorer

Citation for published version (APA):

S R, M. N., Hewitt, R., Monks, P., \& Morrall, P. (2020). Asymptotics of Coupled Reaction-Diffusion Fronts with Multiple Static and Diffusing Reactants: Uranium Oxidation in Water Vapor. SIAM J. Appl. Math., 80(5), 2249-2270. https://doi.org/10.1137/19M1309791

\section{Published in:}

SIAM J. Appl. Math.

\section{Citing this paper}

Please note that where the full-text provided on Manchester Research Explorer is the Author Accepted Manuscript or Proof version this may differ from the final Published version. If citing, it is advised that you check and use the publisher's definitive version.

\section{General rights}

Copyright and moral rights for the publications made accessible in the Research Explorer are retained by the authors and/or other copyright owners and it is a condition of accessing publications that users recognise and abide by the legal requirements associated with these rights.

\section{Takedown policy}

If you believe that this document breaches copyright please refer to the University of Manchester's Takedown Procedures [http://man.ac.uk/04Y6Bo] or contact uml.scholarlycommunications@manchester.ac.uk providing relevant details, so we can investigate your claim.

\section{OPEN ACCESS}




\title{
ASYMPTOTICS OF COUPLED REACTION-DIFFUSION FRONTS WITH MULTIPLE STATIC AND DIFFUSING REACTANTS: URANIUM OXIDATION IN WATER VAPOUR
}

\author{
S.R. MONISHA NATCHIAR*, RICHARD E. HEWITT* ${ }^{*}$, PHILLIP D.D. MONKS ${ }^{\dagger}$, AND \\ PETER MORRALL ${ }^{\dagger}$
}

\section{Abstract.}

Large-time asymptotic solutions for the reaction-diffusion front between one static and one diffusing reactant are known. These states apply to single step reactions with a mean-field reaction rate proportional to $\rho^{m} \alpha^{n}$ (with $m, n \geq 1$ ), where $\rho, \alpha$ are concentrations of the diffusing and static reactants respectively. Such reaction kinetics commonly arise in, for example, simple corrosion models of a porous solid, subject to a diffusing reactant. Here we address a more complex two-step corrosion reaction for oxidation of uranium in a water-vapour environment. In this case, additional complexity arises through a pair of coupled reaction fronts (one with $m=2, n=1$ and the other with $m=3, n=1)$. Furthermore, we allow for material expansion owing to the corrosion process and show that the expected strong dependence of diffusion coefficients on the static reactant distribution is key to explaining experimental observation. In the large-time limit there are four main asymptotic regions, comprising two diffusion layers and two reaction fronts. Asymptotic matching of these regions allows us to construct a large-time solution that gives analytical predictions for the positions of the two propagating fronts, thickness of the diffusion layers and concentration of diffusing species outside of the fronts. This is the first mechanistic model of uranium oxidation in water vapour and (crucially) predicts a thin propagating sub-surface (hydride) layer, as recently observed in atom-probe tomography experiments.

\section{Key words. Reaction-diffusion fronts, asymptotic, uranium oxidation}

AMS subject classifications. 35K57; 35Q74; 35C20

1. Introduction. When uranium is placed in a water vapour environment, an oxide layer grows at exposed surfaces. For moderate exposure times, this oxide layer is adhered to the underlying metal and its rate of growth decreases with time. For longer exposures a secondary stage is found, where cracking and spalling of the oxide layer occurs and the sub-surface oxide interface then propagates into the bulk material with approximately constant velocity. Whilst there are a number of empirical studies (e.g., Baker et al., 1966; Colmenares, 1975; Ritchie, 1984; Winer et al., 1987; McGillivray et al., 1994; Scott et al., 2011) of uranium oxidation in water vapour, to the authors' knowledge there is no well-established mechanistic view of the underlying physical and chemical processes. Furthermore, although simplistic 'models' (e.g., Haycock, 1959) have been fitted to empirical data, these typically have a limited connection with underlying mechanisms, and there is no theoretical/computational model available that ties observable quantities to the parameters that govern the proposed chemical/physical processes.

In a step-change from earlier work, the recent experimental study of Martin et al. (2016) has explicitly analysed the sub-surface structure of uranium following exposure to moist air via atom-probe tomography (APT). Their results provide new insight into the mechanisms of oxidation, most notably by detecting the presence of hydroxide ions in the oxide layer, and for the first time explicitly demonstrating the presence of a hydride layer (approximately 5nm thick after one hour of exposure) between the surface oxide and the bulk metal. Similar experiments using exposure to a $\mathrm{D}_{2} \mathrm{O}$ environment showed deuterides present between the oxide and metal regions, clearly indicating that

* Department of Mathematics, The University of Manchester, Manchester, M13 9PL, UK.

${ }^{\dagger}$ AWE, Aldermaston, Berkshire, RG7 4PR, UK. 
this intermediate layer arises by diffusion from the ambient and a reaction within the bulk metal. The clarification offered by this APT approach prompts us to pursue a new theoretical model, to explain not only the origin of the recently observed hydride layer, but to also predict oxidation rates for moderate exposure times.

Before we introduce a model specific to uranium oxidation, it is instructive to review briefly the broader (reaction-diffusion) literature associated with material corrosion modelling. Slow (diffusive) corrosion of a medium owing to the presence of a reactant is a well-established process. The simplest such mechanism is underpinned by an $A+B \rightarrow C$ chemical reaction between diffusing reactants $A, B$ to produce an inert product $C$. A feature of these systems is the presence of a 'reaction front', which propagates into the medium and separates regions of high concentrations of $A$ and $B$. The reaction is spatially localised at this moving front, leaving behind the inert product $(C)$ as it moves. As this inert diffusion layer grows, flux of reactant to the front is reduced and the process slows, reducing the propagation speed of the front. Typically the diffusion layer thickens as $t^{1 / 2}$, leading to a decreasing $t^{-1 / 2}$ flux of reactant to the front. Systems such as these can often be formulated as a simpler Stefan problem by neglecting the details within the reaction fronts themselves and focusing purely on the larger diffusive lengthscale; a formal link between the reaction-diffusion approach and Stefan formulation was given in the work of Hilhorst et al. (1996).

Theoretical understanding of the reaction fronts in these problems owes much to the work of Gálfi and Rácz (1988), which assumed a one-dimensional mean-field model with second-order reaction kinetics of the form

$$
\frac{\partial a}{\partial t}=D_{a} \frac{\partial^{2} a}{\partial z^{2}}-k a b, \quad \frac{\partial b}{\partial t}=D_{b} \frac{\partial^{2} b}{\partial z^{2}}-k a b .
$$

Here $k a b$ is (dimensionally) a reaction-rate density, but we will retain the more common terminology of 'reaction rate' with $k$ the 'reaction rate constant', where $a, b$ are the concentrations of $A, B$, which have (constant) diffusion coefficients $D_{a, b}>0$. The initial state corresponds to two regions, of non-zero $a$ or $b$, separated by an interface. Much of the focus in this initial work was placed on the case of $D_{a}=D_{b}$ and at large times the reaction rate was shown to take the form

$$
k a b \sim k t^{-2 / 3} F\left(\frac{z-z_{f}}{t^{1 / 6}}\right),
$$

where $z_{f}=O\left(t^{1 / 2}\right)$ is the moving front position and the functional form $(F)$ decays in the far field. The reaction is therefore localised around the $z_{f}$ position, in a layer of thickness $O\left(t^{1 / 6}\right)$ and diminishing in magnitude. A later consideration of the more general case (with $D_{a} \neq D_{b}$ and varied initial concentrations) by Koza (1996) confirmed the assertion of Gálfi and Rácz that the exponents in (1.2) are not dependent on these parameters.

Subsequent computational work (using random walks on a square lattice) by Jiang and Ebner (1990) confirmed the appearance of the predicted lengthscales but also pointed towards a separate class of behaviour when one of the diffusion coefficients is zero. This result was also reinforced by the later analysis of Koza (1997). This issue is significant for the corrosion of a solid, as the reaction is typically between a diffusing species $(A$, say) and a static porous solid $(B)$, hence one of the reactants is immobile and it is appropriate to consider $D_{b}=0$. Notably, this theoretical approach has been validated in the experimental work of Léger et al. (1999), which exposed copper clusters to cupric chloride. 
Theoretical work was continued by Bazant and Stone (2000), who tackled the special case of $D_{b}=0$, whilst also allowing for higher-order reaction kinetics, replacing $k a b$ with $k a^{m} b^{n}$ with $m, n \geq 1$. They demonstrated that propagating front solutions still exist, but the reaction rate (1.2) now becomes

$$
k a^{m} b^{n}=k t^{-\lambda_{1}} F\left(\frac{z-z_{f}}{t^{\lambda_{2}}}\right), \quad \text { where } \quad \lambda_{1}=\frac{m}{m+1}, \lambda_{2}=\frac{m-1}{2(m+1)} .
$$

Given this background for a single reaction-diffusion front between two species, we now return our attention to a more complex corrosion reaction relevant to uranium oxidation. When uranium is exposed to a water-vapour $\left(\mathrm{H}_{2} \mathrm{O}\right)$ environment, it leads to the slow diffusive growth of an oxide layer at the exposed surface. It has been proposed by several authors (e.g., Baker, Less, and Orman, 1966; McGillivray, Geeson, and Greenwood, 1994) and strongly supported by the recent APT work of Martin et al., 2016 that the diffusing reactant in this scenario is the hydroxide ion $\left(\mathrm{OH}^{-}\right)$derived from ambient $\mathrm{H}_{2} \mathrm{O}$ via a surface process. Although the stoichiometric chemical reaction for the corrosion process can be approximated by

$$
\mathrm{U}(\mathrm{s})+2 \mathrm{H}_{2} \mathrm{O}(\mathrm{g}) \longrightarrow \mathrm{UO}_{2}(\mathrm{~s})+2 \mathrm{H}_{2}(\mathrm{~g}),
$$

even early experimental evidence indicated a hydrogen deficit in the gas phase product. As noted above, only recently has the origin of this deficit been directly observed in the form of a sub-surface layer of $\mathrm{UH}_{3}$ in the atom-probe tomography experiments of Martin et al., 2016. A schematic overview of the domain is shown in figure 1, where the surface oxide layer remains separated from the bulk material by the sub-surface hydride region.

In this work, we follow a newly proposed reaction scheme (Glascott and Findlay, 2019), which proceeds with the following elementary steps within the material

$$
\begin{aligned}
\mathrm{UH}_{3}+2 \mathrm{OH}^{-} \stackrel{k_{1}^{*}}{\longrightarrow} \mathrm{UO}_{2}+5 \mathrm{H}^{\bullet}+2 \mathrm{e}^{-}, \\
\mathrm{U}+3 \mathrm{H}^{\cdot} \stackrel{k_{2}^{*}}{\longrightarrow} \mathrm{UH}_{3},
\end{aligned}
$$

where chemical state symbols have been omitted for simplicity. A surface reaction scheme that links with the reactants and products of (1.5) to give an overall reaction of (1.4) is

$$
\begin{aligned}
2 \mathrm{H}_{2} \mathrm{O}+2 \mathrm{O}^{2-}{ }_{l} & \longrightarrow 2{\mathrm{OH}^{-}}_{l}+2 \mathrm{OH}^{-}, \\
2 \mathrm{e}^{-}+2 \mathrm{OH}^{-} & \longrightarrow 2 \mathrm{O}^{2-}{ }_{l}+\mathrm{H}_{2}, \\
2 \mathrm{H}^{\cdot} & \longrightarrow \mathrm{H}_{2},
\end{aligned}
$$

where the subscript $l$ represents lattice-bound species. In this scheme, dissociation of $\mathrm{H}_{2} \mathrm{O}$ occurs at the surface via interaction with an $\mathrm{O}^{2-}{ }_{l}$ anion in (1.6a) to form the diffusing $\mathrm{OH}^{-}$species and a non-diffusing $\mathrm{OH}^{-}$. Formation of the $\mathrm{H}_{2}$ product proceeds according to the two concurrent mechanisms of (1.6b) and (1.6c), with the former consuming electrons produced by the bulk reaction (1.5) and regenerating $\mathrm{O}^{2-}{ }_{l}$. In what follows we will not keep account of the electron production in (1.5), and assume that the surface processes (1.6) can be reduced to simple Dirichlet boundary conditions for concentrations of $\left[\mathrm{OH}^{-}\right]$and $\left[\mathrm{H}^{*}\right]$.

Key to the bulk reaction (producing $\mathrm{UO}_{2}$ from $\mathrm{U}$ ) is that it is a two-step process, with $\mathrm{UH}_{3}$ acting as an intermediate in the formation of $\mathrm{UO}_{2}$. The solid phases $\mathrm{UH}_{3}$, 


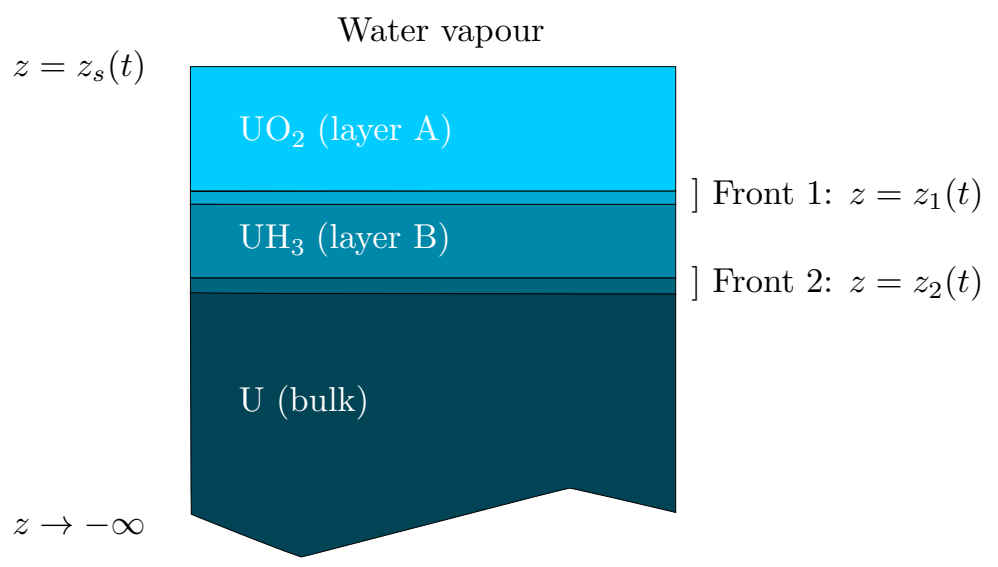

FIG. 1. Schematic diagram of the truncated domain and its typical structure. As the reaction proceeds, $U$ is converted to $U_{3}$ at reaction front 2, whilst $\mathrm{UH}_{3}$ is converted to $\mathrm{UO}_{2}$ at reaction front 1. The two reaction fronts propagate 'downwards' into the bulk material, whilst the upper surface $z_{s}(t)$ moves upwards to accommodate the density reductions associated with the conversion to $\mathrm{UH}_{3}$ and $\mathrm{UO}_{2}$. In the initial state we take $z_{s}(0)=0$.

$\mathrm{U}$ and $\mathrm{UO}_{2}$ (uranium hydride, uranium and uranium dioxide) are "static" whilst the mobile diffusing species are $\mathrm{H}^{*}, \mathrm{OH}^{-}$(hydrogen radicals and hydroxide ions). Although we retain the term "static" (as used by, for example, Bazant and Stone, 2000) for the non-diffusing quantities, we will allow for expansion of the material during corrosion, as necessitated by the substantial decrease in density when $\mathrm{U}$ is converted to $\mathrm{UO}_{2}$ (or $\left.\mathrm{UH}_{3}\right)$.

Our aim in this work is to present a theoretical analysis of a corresponding meanfield model for this more complex reaction system (1.5). In particular we wish to establish if a one-dimensional diffusion-advection-reaction model leads to a sustained propagating intermediate hydride layer (as observed in recent experiments by Martin et al., 2016). To achieve this we first formulate a dimensionless problem (section 2), before presenting some brief numerical solutions in section 3. Our primary approach will be via the analytical/asymptotic methods of section 4 (rather than a purely computational approach), which will allow us to explicitly determine the dominant parameters that govern the corrosion process and, in particular, what sets the thickness of any resulting hydride layer and oxidation rate. We validate the asymptotic theory using large-time results from the numerical solutions in section 5 .

2. Formulation. The analogous system to (1.1) for the more complex reaction (1.5) requires two diffusing species with concentrations $\rho_{1,2}^{*}$ and three "static" (nondiffusing) concentrations $\alpha_{1,2,3}^{*}$, where

$$
\left(\rho_{1}^{*}, \rho_{2}^{*}\right) \equiv\left(\left[\mathrm{OH}^{-}\right],\left[\mathrm{H}^{*}\right]\right), \quad\left(\alpha_{1}^{*}, \alpha_{2}^{*}, \alpha_{3}^{*}\right) \equiv\left(\left[\mathrm{UH}_{3}\right],[\mathrm{U}],\left[\mathrm{UO}_{2}\right]\right)
$$

The index notation is chosen such that the first and second reactions in (1.5) involve reactions between quantities with subscripts 1,2 respectively.

A standard one-dimensional mean-field model is (in terms of dimensional quanti- 
ties, as indicated by the asterisk)

$$
\begin{gathered}
\frac{\partial \rho_{1,2}^{*}}{\partial t^{*}}+\frac{\partial}{\partial z^{*}}\left(v^{*} \rho_{1,2}^{*}\right)=\frac{\partial}{\partial z^{*}}\left(D_{1,2}^{*} \frac{\partial \rho_{1,2}^{*}}{\partial z^{*}}\right)+r_{1,2}^{*}, \\
\frac{\partial \alpha_{1,2,3}^{*}}{\partial t^{*}}+\frac{\partial}{\partial z^{*}}\left(v^{*} \alpha_{1,2,3}^{*}\right)=R_{1,2,3}^{*} .
\end{gathered}
$$

As noted above, whilst the density of $\mathrm{UO}_{2}$ and $\mathrm{UH}_{3}$ are (roughly) comparable, the density of $\mathrm{U}$ is substantially higher and this results in an advection (with velocity $v^{*}$ ) of material in a direction opposite to the propagating reaction fronts.

The nonlinear reaction terms $r_{1,2}^{*}$ and $R_{1,2,3}^{*}$ are defined using the rate law which has a power law structure, that is, proportional to $\rho_{i}^{* m} \alpha_{j}^{* n}$, where $m$ and $n$ are the kinetic orders of the reaction. The corresponding reaction source/sink terms are

$$
\begin{aligned}
\left(r_{1}^{*}, r_{2}^{*}\right) & =\left(-2 k_{1}^{*} \rho_{1}^{* 2} \alpha_{1}^{*}, 5 k_{1}^{*} \rho_{1}^{* 2} \alpha_{1}^{*}-3 k_{2}^{*} \rho_{2}^{* 3} \alpha_{2}^{*}\right), \\
\left(R_{1}^{*}, R_{2}^{*}, R_{3}^{*}\right) & =\left(k_{2}^{*} \rho_{2}^{* 3} \alpha_{2}^{*}-k_{1}^{*} \rho_{1}^{* 2} \alpha_{1}^{*},-k_{2}^{*} \rho_{2}^{* 3} \alpha_{2}^{*}, k_{1}^{*} \rho_{1}^{* 2} \alpha_{1}^{*}\right) .
\end{aligned}
$$

In addition to the diffusing and static reactants, and the advection owing to material expansion, (2.2) also allows for the diffusion coefficients to vary with concentration of the static components, that is

$$
D_{1,2}^{*}=D_{1,2}^{*}\left(\alpha_{1,2,3}^{*}\right) .
$$

This is motivated by the application, where we anticipate substantial changes in diffusivity of $\mathrm{H}^{*}$ in the intermediate $\mathrm{UH}_{3}$ phase, based on the results of Peretz et al. (1976). We will however assume that the diffusivity of mobile reactants 1,2 are independent of $\rho_{1,2}^{*}$ at the (small) concentration levels of interest.

2.1. A dimensionless problem. The reaction is assumed to be driven by a fixed concentration of $\mathrm{OH}^{-}$at the surface of the material, and we denote this constant value as $C^{*}$. Similarly the three static reactants have known maximum concentrations $N_{1,2,3}^{*}$ for pure phases of uranium hydride $\left(4.54 \times 10^{-2} \mathrm{~mol} / \mathrm{cm}^{3}\right)$, uranium $\left(8.03 \times 10^{-2} \mathrm{~mol} / \mathrm{cm}^{3}\right)$ and uranium dioxide $\left(4.06 \times 10^{-2} \mathrm{~mol} / \mathrm{cm}^{3}\right)$ respectively. To non-dimensionalise the concentrations we therefore introduce

$$
\alpha_{1,2,3}^{*}\left(z^{*}, t^{*}\right)=N_{1,2,3}^{*} \alpha_{1,2,3}(z, t), \quad \rho_{1,2}^{*}\left(z^{*}, t^{*}\right)=C^{*} \rho_{1,2}(z, t) .
$$

For the dimensionless timescale $t$ we make use of one of the two reaction rates, then the corresponding lengthscale is obtained from a chosen reference diffusivity value, $D_{r e f}^{*}$ (which we will choose later):

$$
t^{*}=\frac{1}{k_{2}^{*} C^{* 3}} t, \quad z^{*}=\left(\frac{D_{r e f}^{*}}{k_{2}^{*} C^{* 2} N_{2}^{*}}\right)^{\frac{1}{2}} z .
$$

A velocity scale follows directly from the ratio of length and time scales.

The resulting dimensionless system is now

$$
\begin{gathered}
\epsilon\left\{\frac{\partial \rho_{1,2}}{\partial t}+\frac{\partial}{\partial z}\left(v \rho_{1,2}\right)\right\}=\frac{\partial}{\partial z}\left(D_{1,2} \frac{\partial \rho_{1,2}}{\partial z}\right)+r_{1,2}, \\
\frac{\partial \alpha_{1,2,3}}{\partial t}+\frac{\partial}{\partial z}\left(v \alpha_{1,2,3}\right)=R_{1,2,3}
\end{gathered}
$$


where

$$
\begin{aligned}
\left(r_{1}, r_{2}\right) & =\left(-2 k_{1} \rho_{1}^{2} \alpha_{1}, 5 k_{1} \rho_{1}^{2} \alpha_{1}-3 \rho_{2}^{3} \alpha_{2}\right), \\
\left(R_{1}, R_{2}, R_{3}\right) & =\left(\left(\rho_{2}^{3} \alpha_{2}-k_{1} \rho_{1}^{2} \alpha_{1}\right) \frac{N_{2}^{*}}{N_{1}^{*}},-\rho_{2}^{3} \alpha_{2}, k_{1} \rho_{1}^{2} \alpha_{1} \frac{N_{2}^{*}}{N_{3}^{*}}\right),
\end{aligned}
$$

and $D_{1,2}$ are the spatially varying non-dimensional diffusivities. The dimensionless parameter $\epsilon=C^{*} / N_{2}^{*}$ is a measure of the surface concentration of diffusing reactant relative to a reference static reactant, whilst $k_{1}=k_{1}^{*} N_{1}^{*} /\left(k_{2}^{*} N_{2}^{*} C^{*}\right)$ is a relative measure of the two reaction rate constants in (1.5).

In cases of interest, namely oxidation in ambient conditions (room temperature and pressure), the relative concentration of diffusing reactants to static reactants is very small, leading to $\epsilon \ll 1$. For example, using concentrations of water in the ambient gas as a proxy for the surface concentration of $\mathrm{OH}^{-}$(at room temperature) yields $\epsilon \approx 10^{-5}$. We therefore make a quasi-steady approximation for the diffusion processes, but we will retain $k_{1}$ as a parameter. In the subsequent analysis we will demonstrate that it is possible to rescale a reaction front width to account for any arbitrarily large $k_{1}$ associated with the limit of $\epsilon \ll 1$.

The dimensionless quantities $\alpha_{i}$ are in essence volume fractions of the three static components that comprise the bulk material, where $\alpha_{1}+\alpha_{2}+\alpha_{3}=1$. The advection velocity associated with material expansion can be determined via

$$
\frac{\partial v}{\partial z}=\rho_{2}^{3} \alpha_{2}\left(\frac{N_{2}^{*}}{N_{1}^{*}}-1\right)+k_{1} \rho_{1}^{2} \alpha_{1}\left(\frac{N_{2}^{*}}{N_{3}^{*}}-\frac{N_{2}^{*}}{N_{1}^{*}}\right),
$$

as obtained by addition of the $\alpha_{1,2,3}$ equations. In the case of interest $N_{1}^{*} \approx N_{3}^{*}$, whilst $N_{2}^{*} \approx 2 N_{1}^{*}$, which leads to $v$ being an increasing function of $z$ (i.e. the material expands during corrosion).

For the spatially varying diffusion coefficients, we use a linear combination of the pure-phase diffusion coefficients weighted by the respective volume fractions of the static components (Polanowski and Koza, 2006; Ostoja-Starzewski and Schulte, 1996). The effective diffusivities of $\rho_{1}\left(\mathrm{OH}^{-}\right)$and $\rho_{2}\left(\mathrm{H}^{*}\right)$ are therefore

$$
D_{1,2}=\sum_{j=1,2,3} D_{1,2}^{(j)} \alpha_{j},
$$

where the $D_{i}^{(j)}$ are the dimensionless diffusion coefficients for reactant $i$ in a pure phase with $\alpha_{j}=1$. By choosing $D_{r e f}^{*}$ in (2.5b) to be the diffusion coefficient of $\mathrm{OH}^{-}$in $\mathrm{UO}_{2}$, we can take $D_{1}^{(3)}=1$.

Our aim is to evolve (2.6) forward in time (with $\epsilon=0$ ), subject to boundary conditions of

$$
\left.\begin{array}{c}
\rho_{1}=1, \quad \rho_{2}=0 \quad \text { at } \quad z=z_{s}(t) \\
\left(\alpha_{1}, \alpha_{2}, \alpha_{3}\right) \rightarrow(0,1,0), \quad \text { as } z \rightarrow-\infty
\end{array}\right\} \text { for } t \geq 0 .
$$

Here $z_{s}(t)$ is the advecting surface of the expanding solid (such that $\dot{z}_{s}(t)=v\left(z=z_{s}(t)\right.$ ) and $z_{s}(0)=0$ ), which is in contact with the atmosphere, leading to a unit concentration for $\rho_{1}\left(\mathrm{OH}^{-}\right)$by choice of the non-dimensionalisation. The zero condition for $\rho_{2}\left(\mathrm{H}^{*}\right)$ is chosen because outgassing of $\mathrm{H}_{2}$ is observed experimentally, arising from recombination of $\mathrm{H}^{*}$ at the surface of the material $\left(\mathrm{H}_{2}\right.$ itself is only weakly soluble in $\mathrm{UO}_{2}$, see for example Banos et al., 2018). Far from the surface forcing (as $z \rightarrow-\infty$ ), we expect to recover an unreacted solid (with only U present). 

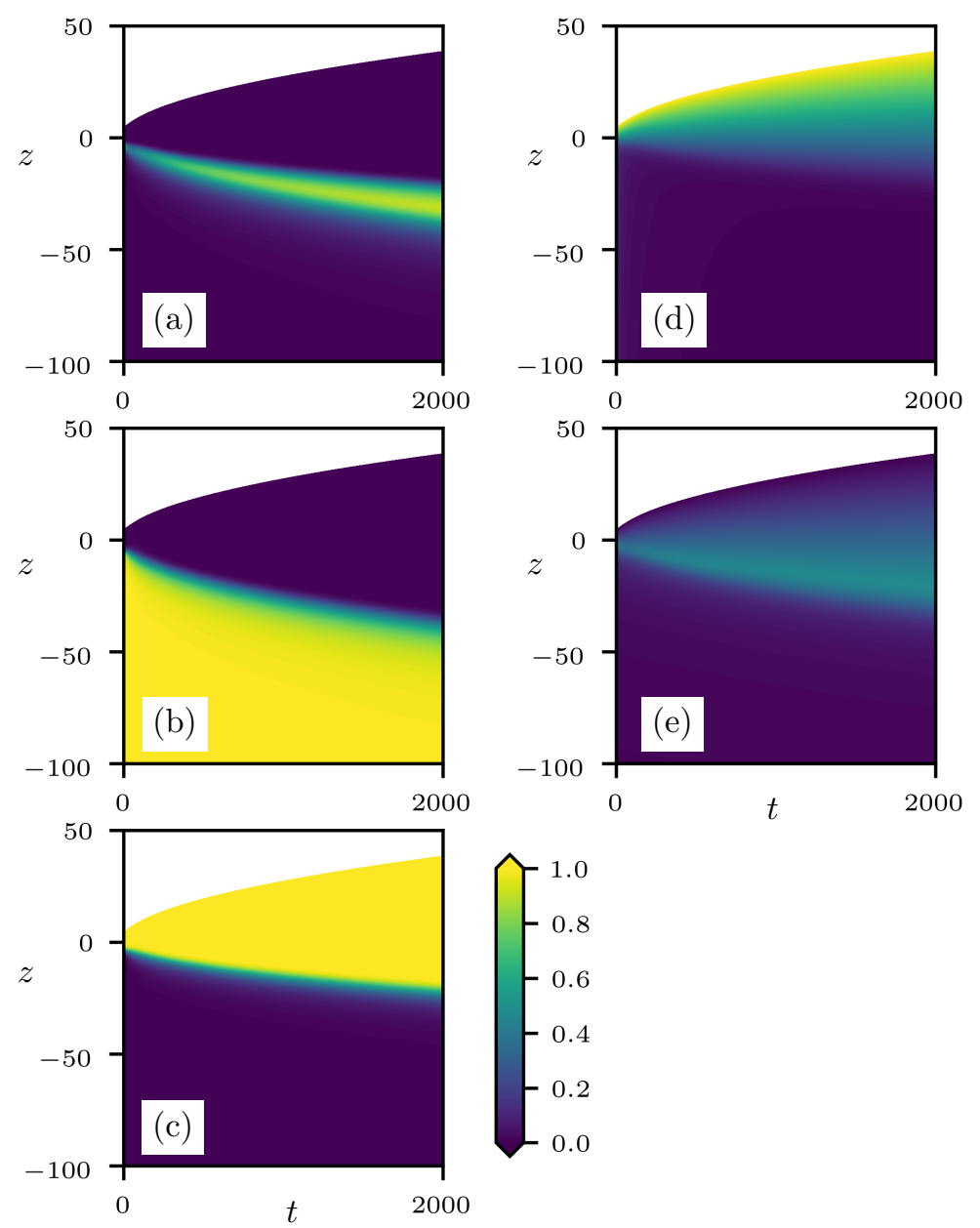

FIG. 2. Development of the dimensionless concentrations in the $z-t$ plane for a numerical solution of (2.6) with $k_{1}=D_{i}^{(j)}=1$. Contours of concentration of each static component are shown in (a) $\alpha_{1}$, (b) $\alpha_{2}$ and (c) $\alpha_{3}$. Contours of concentration for diffusing species are shown in (d) $\rho_{1}$ and $(e) \rho_{2}$.

The initial conditions applied to (2.6) are not critical, beyond there being some initial $\alpha_{1}\left(\mathrm{UH}_{3}\right)$ or $\rho_{2}\left(\mathrm{H}^{*}\right)$, as required to initiate the reaction (1.5). Low levels of hydrogen are found to be present in metals and may exist either as microscopic hydride sites or collocated with other impurities. The precise choice of initial conditions employed in numerical solutions is given in Appendix A.

3. Typical numerical results. Before we develop an asymptotic solution in detail, it is helpful to review some exemplar evolutions of (2.6). Numerical solutions are obtained by a Crank-Nicolson time-marching scheme, applied to a second-order accurate finite-difference discretisation in $z$. To handle the nonlinear nature of the reaction terms, we employ a Newton iteration at each time level. We deal with the growing computational domain by reformulating (2.6) in terms of a transformed coordinate that remains fixed as the solid expands during corrosion; the details of this formulation can 
(a)

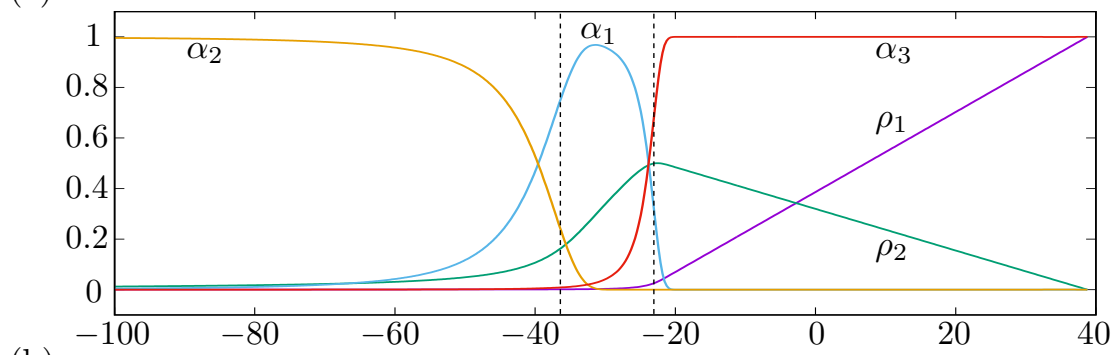

(b)

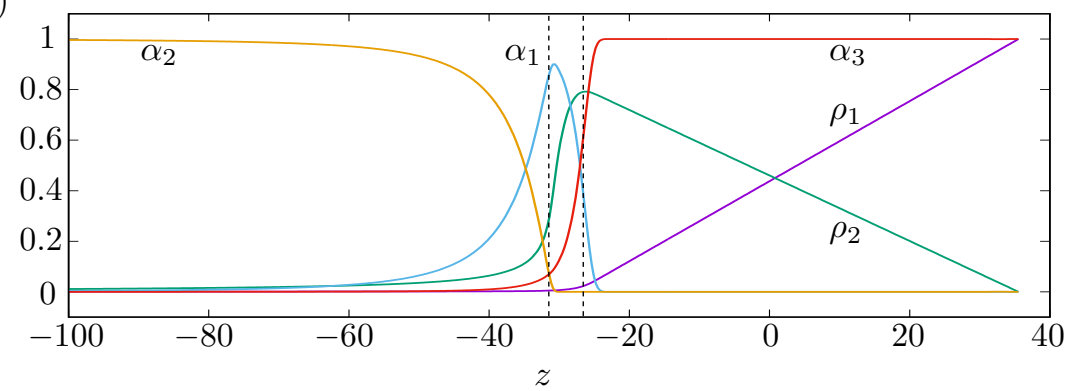

FIG. 3. Dimensionless concentrations of the static $\alpha_{1,2,3}$ and diffusing $\rho_{1,2}$ reactants as measured at $t=2000$ for $k_{1}=10$ and (a) $D_{2}^{(1)}=1$, (b) $D_{2}^{(1)}=0.01 ; D_{i}^{(j)}=1$ for $(i, j) \neq(2,1)$. Reduction of the diffusion coefficient $D_{2}^{(1)}$ is seen to reduce the thickness of the intermediate $\alpha_{1} \neq 0\left(U H_{3}\right)$ layer. Vertical dashed lines denote the corresponding reaction front locations; here determined to be the position of maximum $\rho_{1}^{2} \alpha_{1}$ (right) and $\rho_{2}^{3} \alpha_{2}$ (left).

be found in Appendix A.

In figure 2(a-e) we present contours in the $(z, t)$ plane of $\rho_{1,2}$ and $\alpha_{1,2,3}$, for an evolution of (2.6) with $k_{1}=1$ and $D_{i}^{(j)}=1$ for $i=\{1,2\}, j=\{1,2,3\}$. This corresponds to the reaction rate constants in (1.5) being equal and both diffusing species having equal diffusion coefficients (uniformly throughout the whole domain). The concentrations of the diffusing species $\rho_{1,2}$ are shown on the right-hand side of figure 2. The surface forcing of $\rho_{1}$ is clearly visible whilst $\rho_{2}$ can be seen to attain a maximum value within the domain interior, being produced at a reaction front. Growth of the material surface, away from the $z=0$ initial position, can also be observed in all sub-figures, arising from the density change associated with reaction of higher density metal (volume fraction $\alpha_{2}$ ) to produce lower density hydride and oxide $\left(\alpha_{1,3}\right)$. The left-hand side of figure 2 shows concentrations of the static components, where a growing layer of $\alpha_{3}=1$ is produced near the surface of the domain, separated from the unreacted region $\left(\alpha_{2}=1\right)$ by a propagating (and thickening) layer of $\alpha_{1}=1$. Transitions between these layers occur through a pair of propagating reaction fronts.

Figure 3 shows a snapshot of $\rho_{1,2}$ and $\alpha_{1,2}$ at a large but fixed time value $(t=2000)$. Sub-figure (a) shows the solution for $k_{1}=10, D_{i}^{(j)}=1$, whilst (b) shows the influence of a lower diffusivity of $D_{2}^{(1)}=0.01$ (with all other diffusion coefficients remaining at unity). In both figures the surface of the material has evolved from its initial position at $z=0$, expanding to a higher value owing to the density change induced by the reactions.

Figure 3(a) shows the 'upper' diffusion layer $\left(\alpha_{3}=1\right)$ from $z \approx-20$ to $z \approx 40$, whilst near $z \approx-20$ the first reaction front is associated with a sudden reduction in $\alpha_{3}$ 
and corresponding increase in $\alpha_{1}$. A second reaction front is found at $z \approx-40$, where $\alpha_{1}$ decreases back to zero with an increase in $\alpha_{2}$ to unity. At larger times these two reaction fronts (typically) separate further, leaving a propagating (and widening) layer of $\alpha_{1}=1$ in the intermediate layer.

The influence of the associated parameters in (2.6) will be clarified in the asymptotic descriptions below. At this stage we simply note the role played by a reduction of diffusion coefficient $D_{2}^{(1)}$ on the thickness of the intermediate layer in the figures 3(a) and (b). As this diffusion coefficient is reduced, the two reaction fronts are brought closer together; we will revisit this issue below as it is of some practical concern in uranium oxidation.

4. Asymptotic results. Our goal here is to present an analytical (matched) asymptotic solution to (2.6) in the large-time limit. As part of this process we show how the two reactions generally occur in spatially separated locations, but the full solution remains coupled. Our asymptotic solution not only highlights the dominant physical features, but (analytically) determines the spatial positions of both the reaction fronts, concentrations of the species outside of the fronts, the velocity of the material expansion and the rate of oxidation.

4.1. The general case: $D_{i}^{(j)} \neq 0$. Based on exemplar numerical results, we see that the coupled reaction system (1.5) leads to two propagating reaction fronts that are spatially separated by an intermediate layer (B). In terms of the static component concentrations denoted by $\alpha_{i}$ we see three main regions: layer A (an oxide layer, $\alpha_{3}=1$ ), layer B (a hydride layer, $\alpha_{1}=1$ ) and the remaining unreacted bulk (a metal layer, $\alpha_{2}=1$ ), as shown in the schematic of figure 4 (and figure 1).

These three regions are separated by two propagating reaction fronts, front 1 (between layer $\mathrm{A}$ and layer $\mathrm{B}$ ) where $k_{1} \rho_{1}^{2} \alpha_{1}$ is non-zero and front 2 (between layer $\mathrm{B}$ and the remaining bulk metal) where $\rho_{2}^{3} \alpha_{2}$ is non-zero. It will be seen that these reaction fronts adhere to the scalings of Bazant and Stone (2000), although in this case the reaction front profiles are also influenced by spatially varying diffusivity and material expansion during corrosion, through additional advective transport.

We now present a leading-order matched asymptotic description valid for sufficiently large times. As the reaction develops, the first reaction front propagates into the solid such that its location is

$$
z_{1}(t)=-L_{A} t^{\frac{1}{2}}+\cdots,
$$

with $L_{A}>0$. This "parabolic oxidation" (Ritchie, 1984; McGillivray, Geeson, and Greenwood, 1994), with a square-root time dependence, is observed when the surface oxide layer is not thick enough to crack or spall, and is a characteristic feature of similar reaction-diffusion models (Jiang and Ebner, 1990; Gálfi and Rácz, 1988; Koza, 1996; Bazant and Stone, 2000). The surface displacement due to material expansion must also be of the same form:

$$
z_{s}(t)=L_{s} t^{\frac{1}{2}}+\cdots
$$

Here $L_{s}>0$, signifying that the direction of propagation of the surface is opposite to the direction of the propagating reaction fronts.

4.1.1. Layer A: the oxide layer, $\alpha_{3}=1$. In the oxide layer, $\alpha_{3}=1$ at leading order, with $\alpha_{1,2} \ll 1$, therefore the reaction terms are negligibly small, leaving a simple 


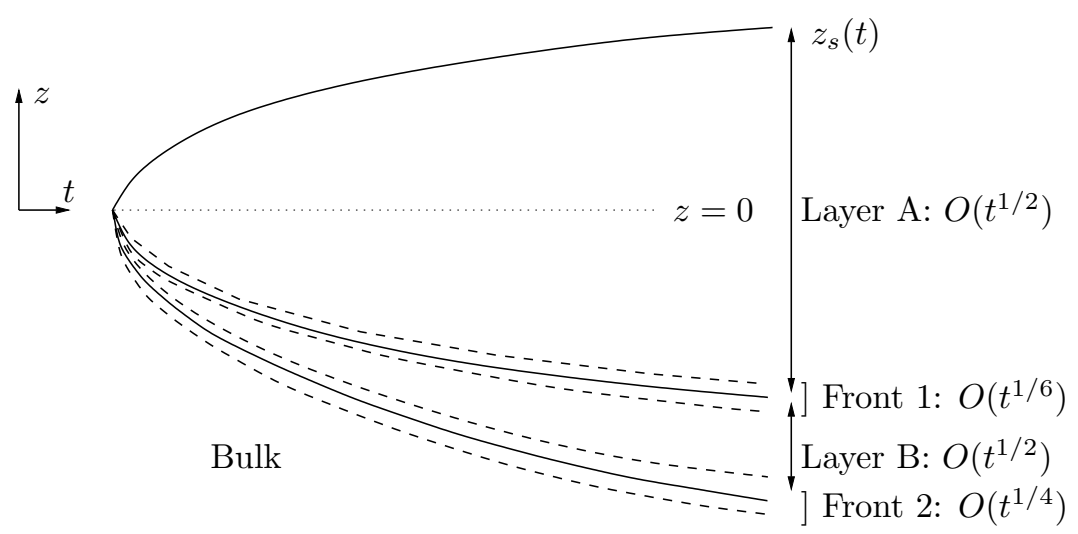

FIG. 4. A schematic of the asymptotic regions in the general case of $D_{2}^{(1)} \neq 0$. The initial surface is located at $z=0$ but the medium expands as the reactions proceed, with the surface identified by $z=z_{s}(t)>0$ for $t>0$. The two reaction fronts are found at $z=z_{1}(t)$ (front 1 ) and $z=z_{2}(t)$ (front 2) and are separated by a diffusion layer (layer B) of thickness $O\left(t^{1 / 2}\right)$.

quasi-steady diffusion of the reactants $\rho_{1,2}$ :

$$
\frac{\partial^{2} \rho_{1,2}}{\partial z^{2}}=0 .
$$

Applying the boundary condition $\rho_{1}=1$ on $z=z_{s}(t)$ and the asymptotic matching condition $\rho_{1} \rightarrow 0$ as $z \rightarrow z_{1}^{+}$provides

$$
\rho_{1} \sim \frac{z-z_{1}(t)}{z_{s}(t)-z_{1}(t)} .
$$

Similarly, applying the boundary condition $\rho_{2}=0$ on $z=z_{s}(t)$ gives

$$
\rho_{2} \sim \mathcal{A}(t)\left(z_{s}(t)-z\right)
$$

at leading order, where $\mathcal{A}(t)$ will be chosen later. Below this simple quasi-steady diffusion layer we find the first reaction front where the term $k_{1} \rho_{1}^{2} \alpha_{1}$ becomes important.

4.1.2. Reaction front 1 . In this inner region we introduce a scaled and shifted coordinate

$$
X=\frac{\left(z-z_{1}(t)\right)}{t^{\lambda}},
$$

where $\lambda$ is determined below, and the associated asymptotic expansion is

$$
\begin{aligned}
\left(\rho_{1}, \rho_{2}\right) & =\left(t^{\lambda-\frac{1}{2}} \bar{\rho}_{1}(X)+\cdots, \gamma+t^{\lambda-\frac{1}{2}} \bar{\rho}_{2}(X)+\cdots\right), \\
\left(\alpha_{1}, \alpha_{3}\right) & =\left(\bar{\alpha}_{1}(X)+\cdots, 1-\bar{\alpha}_{1}(X)+\cdots\right),
\end{aligned}
$$

with $\alpha_{2} \ll 1$.

The $O\left(t^{\lambda-\frac{1}{2}}\right)$ behaviour of $\rho_{1}$ follows from writing the outer solution (4.4) in terms of the inner variable (4.6). An $O(1)$ concentration of $\rho_{2}$ within the first reaction front is consistent with the numerical results of figures 2 and 3, and matching with (4.5) we require

$$
\mathcal{A}(t)=\frac{\gamma}{L_{A}+L_{s}} t^{-\frac{1}{2}}
$$


The reaction rate $k_{1} \rho_{1}^{2} \alpha_{1}$ at leading order remains $O\left(t^{2 \lambda-1}\right)$. So a dominant balance of the $O\left(t^{-\lambda-\frac{1}{2}}\right)$ diffusion term with the reaction requires $\lambda=1 / 6$, a result which is consistent with the scalings presented in Bazant and Stone (2000) for the stoichiometric coefficients associated with the first reaction in (1.5). However, in this case we also have an additional advection velocity in the reaction front associated with the material expansion. From (2.6e), the appropriate scaling for this advection velocity is

$$
v=t^{-\frac{1}{2}} \bar{v}(X)+\cdots,
$$

which gives the following governing (leading-order) equations in this first reaction front:

$$
\begin{aligned}
\frac{\mathrm{d}}{\mathrm{d} X}\left(\bar{D}_{1} \frac{\mathrm{d} \bar{\rho}_{1}}{\mathrm{~d} X}\right) & =2 k_{1} \bar{\rho}_{1}^{2} \bar{\alpha}_{1}, \\
\frac{\mathrm{d}}{\mathrm{d} X}\left(\bar{D}_{2} \frac{\mathrm{d} \bar{\rho}_{2}}{\mathrm{~d} X}\right) & =-5 k_{1} \bar{\rho}_{1}^{2} \bar{\alpha}_{1}, \\
\frac{1}{2} L_{A} \frac{\mathrm{d} \bar{\alpha}_{1}}{\mathrm{~d} X}+\frac{\mathrm{d}\left(\bar{v} \bar{\alpha}_{1}\right)}{\mathrm{d} X} & =-k_{1} \bar{\rho}_{1}^{2} \bar{\alpha}_{1} \frac{N_{2}^{*}}{N_{1}^{*}}, \\
\frac{\mathrm{d} \bar{v}}{\mathrm{~d} X} & =k_{1} \bar{\rho}_{1}^{2} \bar{\alpha}_{1}\left(\frac{N_{2}^{*}}{N_{3}^{*}}-\frac{N_{2}^{*}}{N_{1}^{*}}\right),
\end{aligned}
$$

where

$$
\bar{D}_{1,2}=D_{1,2}^{(1)} \bar{\alpha}_{1}+D_{1,2}^{(3)}\left(1-\bar{\alpha}_{1}\right)
$$

and (as noted above) $D_{1}^{(3)}=1$ by our choice of a reference diffusivity.

This system determines the concentration profiles within the reaction front, subject to matching with layer $\mathrm{A}$

$$
\frac{\mathrm{d} \bar{\rho}_{1}}{\mathrm{~d} X} \sim \frac{1}{L_{s}+L_{A}}, \quad \frac{\mathrm{d} \bar{\rho}_{2}}{\mathrm{~d} X} \sim-\frac{\gamma}{L_{s}+L_{A}}, \quad \bar{\alpha}_{1} \rightarrow 0 \quad \text { as } \quad X \rightarrow \infty,
$$

and recovery of a hydride layer in layer B, with

$$
\bar{\rho}_{1} \rightarrow 0, \quad \frac{\mathrm{d} \bar{\rho}_{2}}{\mathrm{~d} X} \sim \mu, \quad \bar{\alpha}_{1} \rightarrow 1 \quad \text { as } \quad X \rightarrow-\infty ;
$$

matching conditions for the advection velocity will be considered later. Decay of $\bar{\rho}_{1}$ when approaching layer B (the hydride layer) is consistent with the solutions described in Bazant and Stone (2000), effectively requiring all of the diffusing $\mathrm{OH}^{-}$to be consumed at this reaction front, which also agrees with the experimental observations of Martin et al. (2016). In addition, for this coupled reaction we must have a linear (or constant) behaviour for $\bar{\rho}_{2}$ in this same limit because the reaction terms are spatially localised to the reaction front. At this stage the coefficient $\mu$ in (4.10g) is unknown, but will be determined below.

The concentration profiles in this reaction front can be obtained by solving the system (4.10) numerically, subject to the matching constraints as $X \rightarrow \pm \infty$. We present these results later as a check against the full numerical integration of (2.6). However, to make progress on a complete asymptotic solution, we can simply integrate across this reaction front to obtain matching conditions for layer A and layer B. In 
particular, from (4.10) we find that

$$
\begin{gathered}
{\left[\bar{D}_{1} \frac{\mathrm{d} \bar{\rho}_{1}}{\mathrm{~d} X}\right]_{-\infty}^{+\infty}=2 S_{1}, \quad\left[\bar{D}_{2} \frac{\mathrm{d} \bar{\rho}_{2}}{\mathrm{~d} X}\right]_{-\infty}^{+\infty}=-5 S_{1},} \\
{\left[\frac{1}{2} L_{A} \bar{\alpha}_{1}\right]_{-\infty}^{+\infty}+\left[\bar{v} \bar{\alpha}_{1}\right]_{-\infty}^{+\infty}=-S_{1} \frac{N_{2}^{*}}{N_{1}^{*}}, \quad[\bar{v}]_{-\infty}^{+\infty}=S_{1}\left(\frac{N_{2}^{*}}{N_{3}^{*}}-\frac{N_{2}^{*}}{N_{1}^{*}}\right) .}
\end{gathered}
$$

Here $S_{1}$ is the integral

$$
S_{1} \equiv k_{1} \int_{-\infty}^{\infty} \bar{\rho}_{1}^{2} \bar{\alpha}_{1} \mathrm{~d} X
$$

which is proportional to the (total) sink of diffusing reactant throughout the first reaction front. The integral remains finite as the reaction rate in the integrand remains localised within the reaction front.

Using the far-field behaviour (see (4.10f) and (4.10g)), and eliminating $S_{1},(4.11)$ reduces to

$$
\begin{gathered}
\mu=\frac{1}{D_{2}^{(1)}}\left(\frac{5-2 \gamma D_{2}^{(3)}}{2\left(L_{s}+L_{A}\right)}\right), \\
\bar{v}(-\infty)=\frac{N_{2}^{*}}{N_{1}^{*}} \frac{1}{2\left(L_{s}+L_{A}\right)}-\frac{L_{A}}{2}, \\
\bar{v}(+\infty)=\frac{N_{2}^{*}}{N_{3}^{*}} \frac{1}{2\left(L_{s}+L_{A}\right)}-\frac{L_{A}}{2} .
\end{gathered}
$$

In this formulation we have retained $k_{1}$ as a parameter in the reaction front equations. However it should be noted that the system can be transformed to take the same form but with $k_{1}$ replaced by unity through the substitutions $X \rightarrow k_{1}^{-1 / 3} X$ and $\bar{\rho}_{1,2} \rightarrow k_{1}^{-1 / 3} \bar{\rho}_{1,2}$. The value of $k_{1}$, whilst influencing the thickness of this reaction front, has no impact on the matching conditions that arise from (4.12).

4.1.3. Layer B: the hydride layer, $\alpha_{1}=1$. The hydride layer is where $\alpha_{1}=1$ and exists as an intermediate phase in the reaction scheme (1.5). In this region there is no significant amount of $\mathrm{OH}^{-}$and therefore $\rho_{1}$ is small.

In reaction front 1 (as described above) the second diffusing reactant $\left(\mathrm{H}^{*}\right)$ is generated as a product, with a peak (as yet undetermined) concentration of $\gamma$ in that region. Owing to the quasi-steady nature of the diffusing reactants, the concentration of $\mathrm{H}^{*}$ remains linear in layer $B$, which (on matching with reaction front 1 ) results in

$$
\rho_{2}=\gamma+\frac{1}{D_{2}^{(1)}}\left(\frac{5-2 \gamma D_{2}^{(3)}}{2\left(L_{s}+L_{A}\right)}\right)\left(z-z_{1}\right) t^{-\frac{1}{2}}+\cdots .
$$

The second reaction front is found where $\rho_{2}=o(1)$, which occurs at $z=z_{2}(t)$ where

$$
z_{2}(t)=z_{1}(t)-L_{B} t^{\frac{1}{2}}
$$

with

$$
L_{B}=\frac{2 \gamma\left(L_{s}+L_{A}\right) D_{2}^{(1)}}{5-2 \gamma D_{2}^{(3)}} .
$$


At this point we do not know the location of the first reaction front (via the constant $L_{A}$ ), the expanding material surface $\left(L_{s}\right)$, the peak concentration of $\rho_{2}(\gamma)$ or the location of the second reaction front $\left(L_{B}\right)$. However we can form a closed system of algebraic equations for all four of these quantities by an analysis of the second reaction front and matching to this layer B solution.

4.1.4. Reaction Front 2. The second reaction front can be approached in the same manner as the first, with the inner coordinate

$$
Y=\frac{z-z_{2}}{t^{\frac{1}{4}}} .
$$

Here the $1 / 4$ power scaling is arrived at by a dominant balance of the second reaction in (1.5) with diffusion.

The corresponding asymptotic expansion in this propagating front is

$$
\begin{aligned}
\rho_{2} & =t^{-\frac{1}{4}} \hat{\rho}_{2}(Y)+\cdots, \\
\left(\alpha_{1}, \alpha_{2}\right) & =\left(1-\hat{\alpha}_{2}(Y)+\cdots, \hat{\alpha}_{2}(Y)+\cdots\right), \\
v & =t^{-\frac{1}{2}} \hat{v}(Y)+\cdots,
\end{aligned}
$$

as there is no leading-order oxide $\left(\mathrm{UO}_{2}\right)$ present nor is there any diffusing $\mathrm{OH}^{-}$, and hence both $\alpha_{3}$ and $\rho_{1}$ can be neglected.

This leads to the following (leading-order) equations in the second reaction front:

$$
\begin{aligned}
\frac{\mathrm{d}}{\mathrm{d} Y}\left(\hat{D}_{2} \frac{\mathrm{d} \hat{\rho}_{2}}{\mathrm{~d} Y}\right) & =3 \hat{\rho}_{2}^{3} \hat{\alpha}_{2}, \\
\frac{1}{2}\left(L_{A}+L_{B}\right) \frac{\mathrm{d} \hat{\alpha}_{2}}{\mathrm{~d} Y}+\frac{\mathrm{d}\left(\hat{v} \hat{\alpha}_{2}\right)}{\mathrm{d} Y} & =-\hat{\rho}_{2}^{3} \hat{\alpha}_{2}, \\
\frac{\mathrm{d} \hat{v}}{\mathrm{~d} Y} & =\hat{\rho}_{2}^{3} \hat{\alpha}_{2}\left(\frac{N_{2}^{*}}{N_{1}^{*}}-1\right),
\end{aligned}
$$

where

$$
\hat{D}_{2}=D_{2}^{(1)}\left(1-\hat{\alpha}_{2}\right)+D_{2}^{(2)} \hat{\alpha}_{2} .
$$
$\mathrm{B}$

This system determines the concentration profiles, subject to matching with layer

$$
\frac{\mathrm{d} \hat{\rho}_{2}}{\mathrm{~d} Y} \sim \frac{1}{D_{2}^{(1)}}\left(\frac{5-2 \gamma D_{2}^{(3)}}{2\left(L_{s}+L_{A}\right)}\right), \quad \hat{\alpha}_{2} \rightarrow 0, \quad \text { as } \quad Y \rightarrow \infty,
$$

and recovery of an unreacted solid when moving into the bulk of the metal

$$
\hat{\rho}_{2} \rightarrow 0, \quad \hat{\alpha}_{2} \rightarrow 1, \quad \text { as } \quad Y \rightarrow-\infty .
$$

Again we will consider matching conditions for the advection velocity as a final step below.

We postpone a numerical solution of (4.17) until later, and instead note that the same integrated approach to this reaction front provides

$$
\begin{gathered}
{\left[\hat{D}_{2} \frac{\mathrm{d} \hat{\rho}_{2}}{\mathrm{~d} Y}\right]_{-\infty}^{+\infty}=3 S_{2}, \quad \frac{1}{2}\left(L_{A}+L_{B}\right)\left[\hat{\alpha}_{2}\right]_{-\infty}^{+\infty}+\left[\hat{v} \hat{\alpha}_{2}\right]_{-\infty}^{\infty}=-S_{2},} \\
{[\hat{v}]_{-\infty}^{\infty}=S_{2}\left(\frac{N_{2}^{*}}{N_{1}^{*}}-1\right)}
\end{gathered}
$$




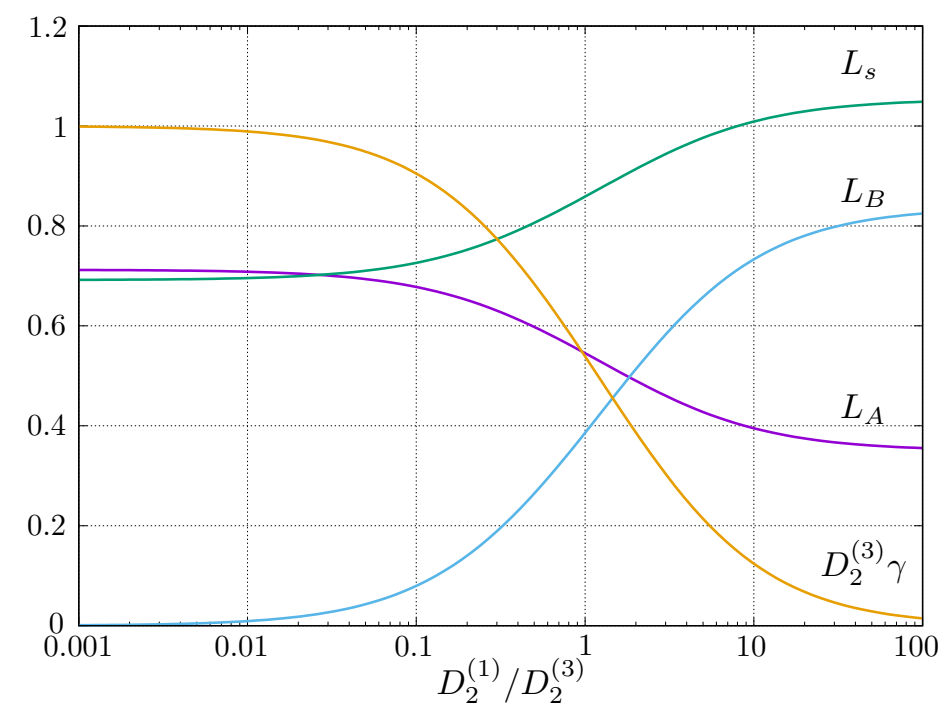

FIG. 5. Dependence of $L_{A}, L_{B}, L_{s}$ and $D_{2}^{(3)} \gamma$ on the ratio of diffusivities $D_{2}^{(1)} / D_{2}^{(3)}$. Given $L_{A, B, s}$, the positions of the two reaction fronts and the expanding surface of the solid are known via (4.1), (4.14a) and (4.2) respectively. Here these results use $N_{1}^{*} / N_{3}^{*} \approx 1.12$ and $N_{2}^{*} / N_{1}^{*} \approx 1.76$, as appropriate for the uranium oxidation example.

where $S_{2}$ is a (finite) integral of the (localised) reaction rate $\hat{\rho}_{2}^{3} \hat{\alpha}_{2}$ over the whole reaction front.

From these depth integrated equations we deduce that

$$
\begin{aligned}
& \frac{1}{2}\left(L_{A}+L_{B}\right)=\frac{1}{6}\left(\frac{5-2 \gamma D_{2}^{(3)}}{L_{s}+L_{A}}\right), \\
& \hat{v}(+\infty)=\left(\frac{N_{2}^{*}}{N_{1}^{*}}-1\right)\left(\frac{L_{A}+L_{B}}{2}\right),
\end{aligned}
$$

as $\hat{v}(-\infty)=0$ in the uncorroded metal.

In this asymptotic description we have four constants: $L_{s}$ which determines the surface position of the expanding solid, $L_{A, B}$ which locate the first and second reaction fronts respectively, and $\gamma$ which is a measure of $\mathrm{H}^{*}$ concentration in the first reaction front. As a final step we require that the advection velocities match across layer $\mathrm{B}$, that is $\bar{v}(-\infty)=\hat{v}(+\infty)$ in (4.12) and (4.20) (because the advection is spatially varying only in the reaction fronts whilst constant in layer B) and using (4.2) we have $\bar{v}(+\infty)=L_{s} / 2$ (as the surface is advected in the expansion process). From this we find that

$$
\begin{gathered}
\frac{N_{2}^{*}}{N_{1}^{*}} \frac{1}{\left(L_{s}+L_{A}\right)}-L_{A}=\left(\frac{N_{2}^{*}}{N_{1}^{*}}-1\right)\left(L_{A}+L_{B}\right), \\
\frac{N_{2}^{*}}{N_{3}^{*}} \frac{1}{\left(L_{s}+L_{A}\right)}-L_{A}=L_{s},
\end{gathered}
$$

which together with (4.14b) and (4.20a) closes the algebraic system.

After some algebra, the solution for $\gamma$ (the leading-order concentration of $\mathrm{H}^{*}$ in the 
first reaction front) reduces to

$$
8 D_{2}^{(3)} \gamma=14+6 \frac{D_{2}^{(1)}}{D_{2}^{(3)}} \frac{N_{1}^{*}}{N_{3}^{*}}-\left\{\left(14+6 \frac{D_{2}^{(1)}}{D_{2}^{(3)}} \frac{N_{1}^{*}}{N_{3}^{*}}\right)^{2}-160\right\}^{\frac{1}{2}}
$$

and the solutions for $L_{A}, L_{B}$ and $L_{s}$ then follow directly. These three coefficients, which determine the positions of the two reaction fronts and the material surface, are only dependent on the ratio $D_{2}^{(1)} / D_{2}^{(3)}$, for fixed values of $N_{i}^{*}$. Furthermore, the value of $k_{1}$, which fixes the relative reaction rate constants in (1.5) has no impact on this leading-order description; it only serves to determine the thickness of the first reaction front as noted in section 4.1.2.

In terms of the application to uranium corrosion, the values of $N_{i}^{*}$ are well known $\left(N_{1}^{*} / N_{3}^{*} \approx 1.12\right.$ and $\left.N_{2}^{*} / N_{1}^{*} \approx 1.76\right)$, but the diffusivity values for $\mathrm{H}^{\cdot}$ in $\mathrm{UH}_{3}\left(D_{2}^{(1)}\right)$ and in $\mathrm{UO}_{2}\left(D_{2}^{(3)}\right)$ are much less established. With this in mind, we show in figure 5 the dependence of these quantities on the diffusivity ratio. Of particular note is the limit $D_{2}^{(1)} / D_{2}^{(3)} \ll 1$, where $L_{B} \rightarrow 0$ and $\gamma \rightarrow 1 / D_{2}^{(3)}$. This indicates that the thickness of the intermediate (hydride) layer is small in this limit, bringing the two propagating reaction fronts closer together at any fixed time value.

4.2. A special case: $D_{2}^{(1)}=0, D_{2}^{(3)} \neq 0$. As $D_{2}^{(1)} / D_{2}^{(3)} \rightarrow 0$ the structure of figure 4 must be modified to account for the fact that $L_{B} \rightarrow 0$ (the leading-order thickness of layer B is zero). From (4.10) in reaction front 1, we see that the governing equation for $\bar{\rho}_{2}$ is independent of those for $\left(\bar{\rho}_{1}, \bar{\alpha}_{1}, \bar{v}\right)$, i.e. the coupling is only one way for these quantities. Furthermore, the ratio $D_{2}^{(1)} / D_{2}^{(3)}$ only influences $\bar{\rho}_{2}$.

From (4.10), applying only the conditions for matching with layer A for $\bar{\rho}_{2}$, we find that

$$
\frac{\mathrm{d} \bar{\rho}_{2}}{\mathrm{~d} X}=\frac{1}{\bar{D}_{2}(X)}\left\{\frac{5-2 \gamma D_{2}^{(3)}}{2\left(L_{s}+L_{A}\right)}-5 k_{1} \int_{-\infty}^{X} \bar{\rho}_{1}^{2}(\bar{X}) \bar{\alpha}_{1}(\bar{X}) \mathrm{d} \bar{X}\right\},
$$

where $\bar{D}_{2}(X)$ is as given in (4.10e).

In our previous analysis of the case $D_{2}^{(1)}>0, \bar{D}_{2}(-\infty)=D_{2}^{(1)}$ and the gradient of $\bar{\rho}_{2}$ remains finite for large negative values of $X$. However, when $D_{2}^{(1)}=0, \bar{D}_{2}(X) \ll 1$ as $X \rightarrow-\infty$ and we have to address the limiting behaviour to determine what sets the thickness of the intermediate (hydride) layer in this case.

As $X \rightarrow-\infty$, the asymptotic behaviour of reaction front 1 , as described by (4.10) is

$$
\begin{gathered}
\bar{\rho}_{1}=a X^{-2}+\cdots, \quad \bar{\alpha}_{1}=1+b X^{-3}+\cdots, \\
\bar{\alpha}_{3}=-b X^{-3}+\cdots, \quad \bar{v}=\bar{v}(-\infty)-c X^{-3}+\cdots,
\end{gathered}
$$

where the constants $a, b, c$ are all positive and $\bar{v}(-\infty)$ is again given by (4.12b) since $\left(\bar{\rho}_{1}, \bar{\alpha}_{1}\right) \rightarrow(0,1)$ in this limit (as before). It is straightforward to show that

$$
a=\frac{3 D_{1}^{(1)}}{k_{1}}, \quad b=\frac{6 D_{1}^{(1)^{2}}\left(N_{2}^{*} / N_{3}^{*}\right)^{\frac{3}{2}}}{k_{1}\left(N_{2}^{*} / N_{1}^{*}\right)}, \quad c=\frac{3 D_{1}^{(1)^{2}}}{k_{1}}\left(\frac{N_{2}^{*}}{N_{3}^{*}}-\frac{N_{2}^{*}}{N_{1}^{*}}\right),
$$

on writing $L_{A}+L_{s}=\left(N_{2}^{*} / N_{3}^{*}\right)^{1 / 2}$, which follows from matching the advection velocity of the first reaction front with layer $\mathrm{A}$ via $\bar{v}(+\infty)=-L_{s} / 2$. 
From the general solution (4.23), using

$$
\bar{D}_{2}(X) \sim D_{2}^{(3)}\left(-b X^{-3}\right),
$$

the corresponding behaviour for $\rho_{2}$ in reaction front 1 is

$$
\rho_{2}=\gamma-\left(\frac{3 X^{4}}{8 b D_{2}^{(3)}}\left(\frac{N_{3}^{*}}{N_{2}^{*}}\right)^{\frac{1}{2}}+O(X)\right) t^{-\frac{1}{3}}+\cdots,
$$

as $X \rightarrow-\infty$. In the previous case $\gamma$ was determined by matching with the second reaction front, but here we will assume consistency with the previous analysis as $D_{2}^{(1)} \rightarrow$ 0 , giving $\gamma=1 / D_{2}^{(3)}$.

This expansion for $\rho_{2}$ disorders when $|X|=O\left(t^{1 / 12}\right)$ or, in terms of the original (outer) coordinate, when

$$
z=z_{2}(t) \equiv z_{1}(t)-\left(\frac{8 b}{3}\left(\frac{N_{2}^{*}}{N_{3}^{*}}\right)^{\frac{1}{2}}\right)^{\frac{1}{4}} t^{\frac{1}{4}}
$$

which is the (leading-order) location of the second reaction front in this special case. This now gives us the large-time prediction of the thickness of the intermediate (hydride) layer $z_{1}(t)-z_{2}(t)$.

In the general case (with non-zero $D_{2}^{(1)}$ ) the two reaction fronts are separated by an $O\left(D_{2}^{(1)} t^{\frac{1}{2}}\right)$ diffusion layer. When $D_{2}^{(1)}=0$, this separation is reduced to $O\left(t^{\frac{1}{4}} k_{1}^{-\frac{1}{4}}\right)$ and no intermediate diffusion layer exists in the leading-order description, but the two reaction fronts stay spatially isolated.

5. A comparison with the full numerical solution. The large-time asymptotic behaviour described in section 4 is qualitatively consistent with the preliminary numerical results of section 3 , however we now check the quantitative details. To begin with, we note that the reaction front solutions to (4.10) and (4.17) are invariant under the translations of $X \rightarrow X+X_{0}$ and $Y \rightarrow Y+Y_{0}$ respectively for constants $X_{0}, Y_{0}$, corresponding to higher-order corrections to the front locations. For comparison of the leading-order predictions with numerical results we are therefore free to define $z_{1}(t)$ as the position at which the first reaction rate,

$$
\mathcal{R}_{1}(z, t)=k_{1} \rho_{1}^{2}(z, t) \alpha_{1}(z, t),
$$

attains its maximum value. Similarly $z_{2}(t)$ will be chosen such that

$$
\mathcal{R}_{2}(z, t)=\rho_{2}^{3}(z, t) \alpha_{2}(z, t)
$$

is maximised.

The asymptotic predictions are that $\left(z_{s}, z_{1}, z_{2}\right) \sim\left(L_{s},-L_{A},-\left(L_{A}+L_{B}\right)\right) t^{1 / 2}$ where the constants $L_{s}, L_{A}, L_{B}$ are as given in figure 5 . We begin by comparing the rescaled thickness of layer A, given by $\left(z_{s}-z_{1}\right) t^{-1 / 2}$ with the asymptotic prediction of $L_{s}+L_{A}$ in figure 6(a). A similar calculation can be made for the thickness of layer B by comparing $\left(z_{1}-z_{2}\right) t^{-1 / 2}$ with $L_{B}$, as shown in figure $6(\mathrm{~b})$. From the asymptotic results of section 4 we know that the value of $k_{1}$ does not affect the $t \gg 1$ leading-order predictions, but the value of $D_{2}^{(1)}$ does affect the value of $L_{B}$ (as shown in figure 5). 

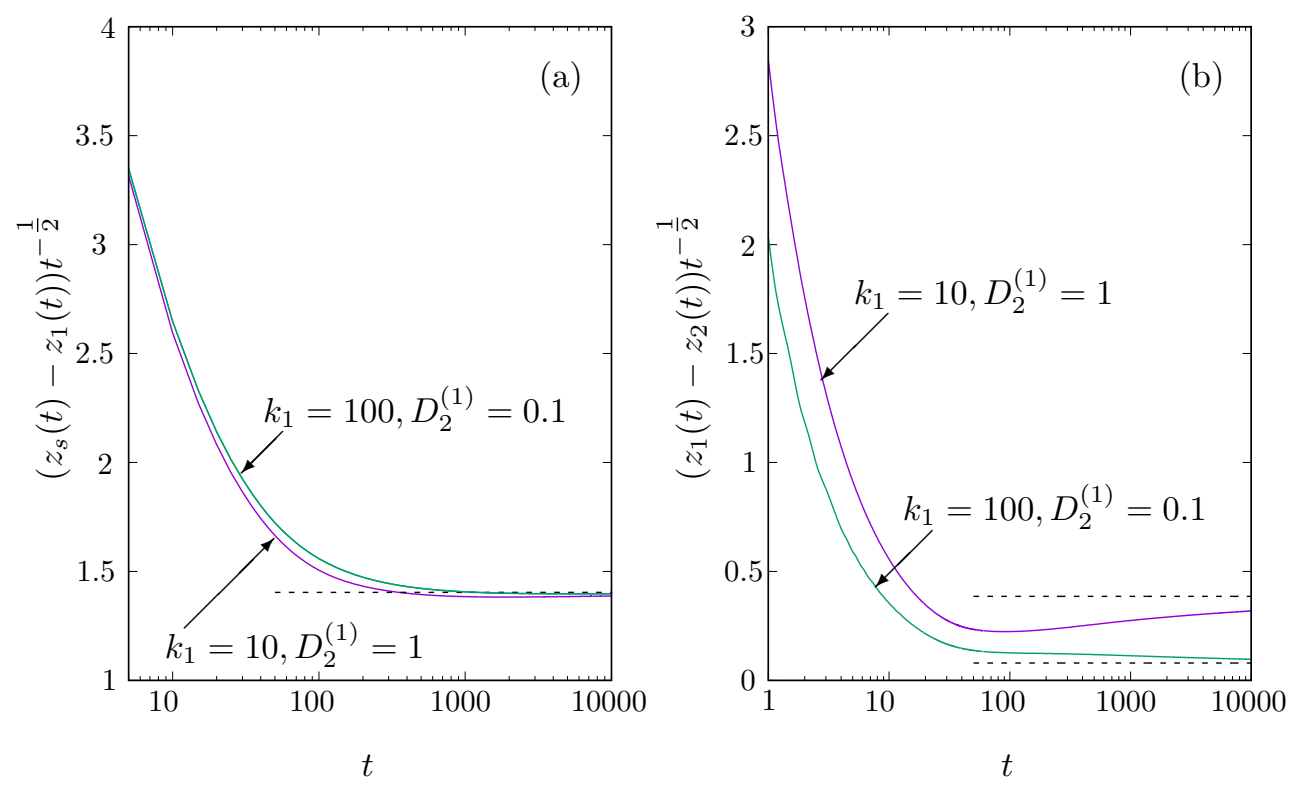

FIG. 6. A comparison of rescaled time-marched numerical solutions to (2.6) (solid lines) with the large-time asymptotic predictions (dashed lines). The dashed line represents (a) $L_{A}+L_{s}$ and (b) $L_{B}$. This confirms that the predicted size of the diffusion layers $A$ and $B$ agree with the full numerical solution for large times.

Figure 6 demonstrates agreement between the full numerical solution and the predicted behaviour for sufficiently large values of $t$ for a range of parameter values.

Within the reaction fronts themselves, we can compare numerical solutions with the leading-order asymptotic profiles obtained from solution of (4.10) and (4.17). To achieve this we take the numerical data, and present it as a function of the reaction front coordinates $X=\left(z-z_{1}\right) t^{-1 / 6}$ and $Y=\left(z-z_{2}\right) t^{-1 / 4}$. Comparisons are then made with $\left(\bar{\alpha}_{1}, \bar{v}_{1}, \overline{\mathcal{R}}_{1}\right)$ in figure $7(\mathrm{~b})$ for reaction front 1 , and $\left(\hat{\alpha}_{2}, \hat{v}_{2}, \hat{\mathcal{R}}_{2}\right)$ in figure $7(\mathrm{a})$ for reaction front 2 . Here $\overline{\mathcal{R}}_{1}$ is the equivalent expression to $\mathcal{R}_{1}$ but using $\bar{\rho}_{1}$ and $\bar{\alpha}_{1}$; similarly for $\hat{\mathcal{R}}_{2}$. Again, there is good agreement between the predicted asymptotic behaviour and the numerical solution of (2.6) obtained at $t=10^{4}$.

Finally, in figure 8 we show the evolution of $z_{1}-z_{2}$ (the thickness of layer B) as a function of time for $D_{2}^{(1)}=1,0.1,0$; with all other values of $D_{i}^{(j)}$ set to unity. The figure also shows the asymptotic predictions: $z_{1}-z_{2}=L_{B} t^{1 / 2}$ (valid for $D_{2}^{(1)}=1,0.1$ ) where $L_{B}$ as a function of $D_{2}^{(1)}$ is as shown in figure 5 . In the case of $D_{2}^{(1)}=0$, we know that $L_{B}=0$ and the modified structure presented in section 4.2 is the appropriate comparison to make for large times, using (4.27). This transition from a layer B thickness of $O\left(t^{1 / 2}\right)$ to a thinner $O\left(t^{1 / 4}\right)$ layer as the diffusion coefficient $D_{2}^{(1)}$ is reduced to zero is seen to be consistent with the full numerical data.

6. Discussion. For the first time, we have developed a mechanistic (reactionadvection-diffusion) model of uranium oxidation in a water-vapour environment. The model is underpinned by a pair of (proposed) reactions that aim to describe uranium corrosion. The model includes advective transport associated with expansion of the material during the corrosion process, variable diffusion coefficients and third/fourthorder reaction kinetics. The (large-time) asymptotic solution (in the general case) 

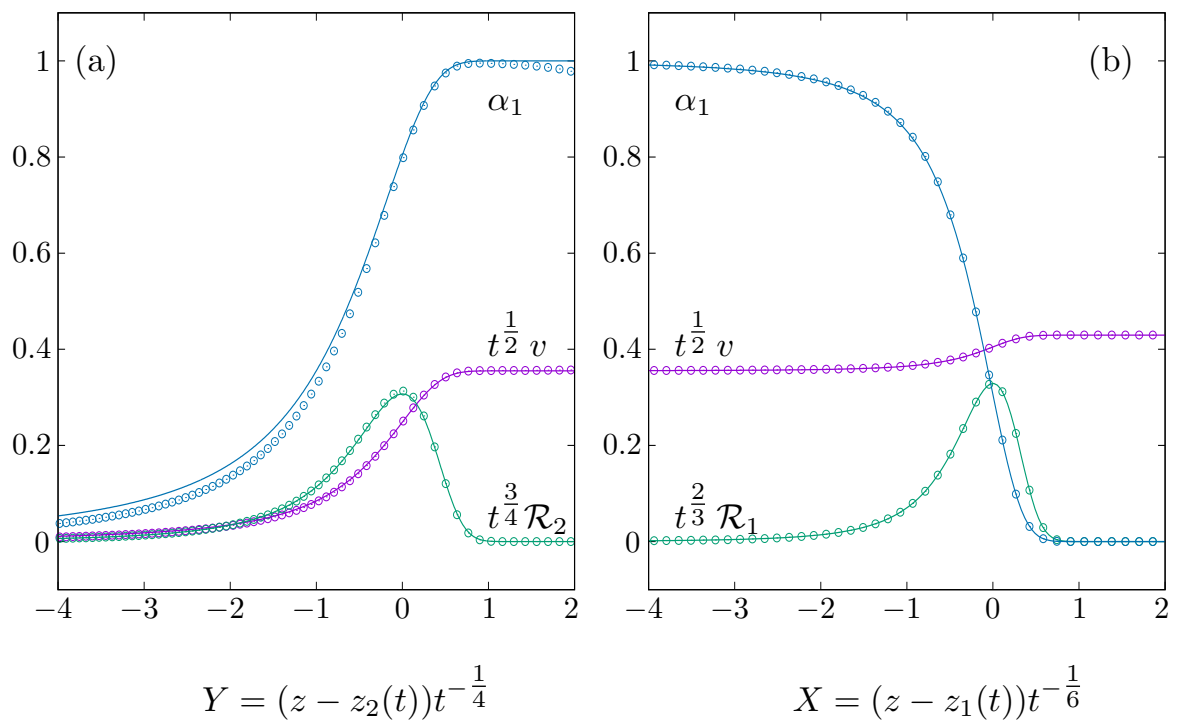

FIG. 7. A comparison of rescaled time-marched numerical solutions to (2.6) (data points) with leading-order asymptotic solutions (solid lines) within (a) reaction front 2 and (b) reaction front 1 for the case of $k_{1}=10, D_{i}^{(j)}=1$ for all $i, j$. The reaction front asymptotic solutions are determined by solution of (a) equations (4.17) and (b) equations (4.10) and the numerical solution is shown for $t=10^{4}$. This confirms that the predictions for the reaction fronts are consistent with numerical solution of the full problem at large times.

reduces to four main regions, comprising two diffusion layers and two reaction fronts. Matching across all four regions provides not just analytical expressions for the overall structure of the material response but also indicates key parameter dependencies.

The locations of the oxide/hydride layers (in the large-time limit) are dominated by the parameter ratio $D_{2}^{(1)} / D_{2}^{(3)}$, as shown in figure 5 . This is equivalent to the corresponding ratio of dimensional values, representing a relative measure of the diffusivity of $\mathrm{H}^{*}$ in $\mathrm{UH}_{3}$ relative to $\mathrm{UO}_{2} . \mathrm{H}^{\bullet}$ and $\mathrm{UH}_{3}$ are both produced and consumed in the twostep reaction (1.5), with $\mathrm{H}^{*}$ arising as a product in reaction front 1 (by consumption of $\mathrm{UH}_{3}$ ) and $\mathrm{UH}_{3}$ arising as a product in reaction front 2 (by consumption of $\mathrm{H}^{*}$ ).

Although the individual values of $L_{s, A, B}$ depend on $D_{2}^{(1)} / D_{2}^{(3)}$, the combination that determines the oxide thickness is $L_{s}+L_{A}=\left(N_{2}^{*} / N_{3}^{*}\right)^{1 / 2}$, which follows from (4.21b). This allows us to predict the (dimensional) oxide thickness to be

$$
\Delta_{\text {oxide }}^{*} \sim\left(D_{\text {ref }}^{*} \frac{C^{*}}{N_{3}^{*}} t^{*}\right)^{1 / 2},
$$

at leading order, where $D_{r e f}^{*}$ is a reference diffusivity (herein chosen as $D_{1}^{(3) *}$, the diffusion coefficient of $\mathrm{OH}^{-}$in $\mathrm{UO}_{2}$ ) and $C^{*}$ is the concentration of $\mathrm{OH}^{-}$at the surface of the material.

Of particular interest is the thickness of layer B (figures 1 and 4), which is an intermediate (hydride) layer as observed for the first time in the recent experimental work of Martin et al. (2016). The dimensionless thickness of this layer is $L_{B} t^{1 / 2}$ at leading order, where (as noted above) $L_{B}$ is determined as a function of $D_{2}^{(1)} / D_{2}^{(3)}$ (figure 5). Based on the experimental data of Peretz et al. (1976), we expect the diffusivity of $\mathrm{H}^{*}$ in $\mathrm{UH}_{3}$ to be substantially smaller than the reference diffusivity (by 


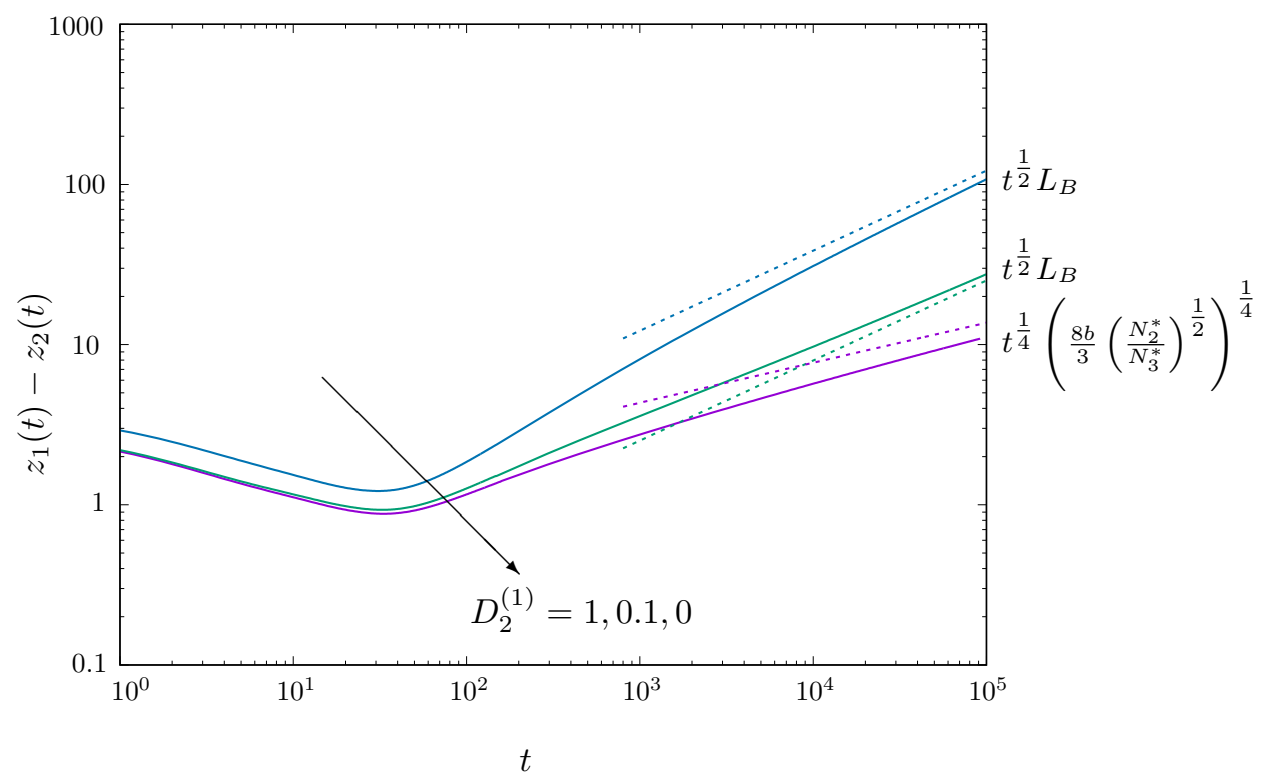

FIG. 8. A comparison of the thickness of layer B, as obtained from time-marched numerical solutions to (2.6) (solid lines) with leading-order $(t \gg 1)$ asymptotic solutions (dashed lines) for the cases of $k_{1}=100, D_{2}^{(1)}=1,0.1,0$ (other values of $D_{i}^{(j)}$ are set to unity). For $D_{2}^{(1)}=1$ and 0.1 the value $L_{B}$ is determined as shown in figure 5, whilst for $D_{2}^{(1)}=0$ the comparison in made with (4.27). This confirms the asymptotic predictions for the thickness of the intermediate layer $B$.

approximately three orders of magnitude) at room temperature. This suggests that the relevant practical limit is $D_{2}^{(1)} \ll D_{2}^{(3)}$, for which

$$
L_{B} \sim \frac{2}{3}\left(\frac{N_{2}^{*}}{N_{3}^{*}}\right)^{1 / 2} \frac{D_{2}^{(1)}}{D_{2}^{(3)}} .
$$

When this is cast into dimensional terms, we conclude that the large-time thickness of the intermediate (hydride) layer is

$$
\Delta_{\text {hydride }}^{*} \sim \frac{2}{3} \frac{D_{2}^{(1)}}{D_{2}^{(3)}} \Delta_{\text {oxide }}^{*},
$$

in the small diffusivity limit of $0<D_{2}^{(1)} / D_{2}^{(3)} \ll 1$. Hence, this reaction-diffusion model offers a mechanism for the sustained, propagating, hydride layer of Martin et al., 2016, and its relative thinness (compared to the length scale of the oxide layer) is shown to be a consequence of weak diffusivity within the hydride layer of the produced $\mathrm{H}^{*}$.

Given that our expectation is for $D_{2}^{(1)}$ to be small, our asymptotic results also explicitly address the case of $D_{2}^{(1)}=0$. In this case the hydride layer (dimensionless) thickness reduces further to $O\left(k_{1}^{-1 / 4} t^{1 / 4}\right)$, where $k_{1}=k_{1}^{*} N_{1}^{*} /\left(k_{2}^{*} N_{2}^{*} C^{*}\right)$.

In our analysis, the local diffusion coefficient was assumed to be a linear weighted combination of the constituent static components, e.g.

$$
D_{1,2}(z, t)=D_{1,2}^{(1)} \alpha_{1}(z, t)+D_{1,2}^{(2)} \alpha_{2}(z, t)+D_{1,2}^{(3)} \alpha_{3}(z, t),
$$

for constants $D_{i}^{(j)}$. This approach has also been taken by (for example) Polanowski and Koza (2006), however it should be noted that any other more complex functional 
dependence of $D_{1,2}$ on $\alpha_{1,2,3}$ will only influence the details of the solutions within the reaction fronts (i.e., figure 7). The large-time conclusions for the thickness of the oxide/hydride layers (when $D_{2}^{(1)}>0$ ) are independent of this choice because one integrates over the reaction fronts to obtain the large-time matching conditions, and these integrated conditions only depend on $D_{i}^{(j)}$ rather than on the precise functional form within the fronts. For the case $D_{2}^{(1)}=0$, the $O\left(t^{1 / 4}\right)$ intermediate layer predicted in section 4.2 would be found for any functional dependence that satisfies $\bar{D}_{2} \sim \bar{\alpha}_{3}$ when $\bar{\alpha}_{3} \ll 1$ within the first reaction front.

A more quantitative comparison with oxide thickness (or mass gain) would be possible with some fitting of parameters, but care must be taken for the oxide to be adherent in experimental work. Any cracking or spalling near the surface will almost certainly lead to faster than $t^{1 / 2}$ growth of the oxide layer. For quantitative application of these asymptotic (rather than computational) results, we require a large time (made dimensionless via the reaction time scale), but not so large that break up of the oxide layer has occurred. A separate analysis (in preparation) is required for the later cracked/spalled-oxide regime, with preliminary results indicating that the sub-surface hydride layer ceases to thicken further as the oxidation rate approaches a constant.

Acknowledgements. The support of AWE plc is gratefully acknowledged by both REH \& SRMN. In addition, SRMN was supported by the UK Engineering and Physical Sciences Research Council (EPSRC). The authors benefited from a number of discussions with J. Petherbridge and R. Harker (AWE) whilst the reaction scheme (1.5) was originally proposed by J. Glascott and I. Findlay (AWE).

\section{Appendix A. Numerical formulation.}

We apply a Howarth-Dorodnitsyn transformation to (2.6), which fixes the moving surface caused by expansion of the material during the reaction process. To achieve this we introduce a material coordinate $\zeta$ defined as

$$
\zeta=\int_{-z_{\infty}}^{z} \varrho \mathrm{d} z \quad \in\left[0, \zeta_{s}\right]
$$

Here $z_{\infty}$ is a (fixed) domain truncation chosen to be sufficiently large to encompass both reaction fronts over the entire timescale of the computation. The quantity $\varrho$ is chosen to be

$$
\varrho=\alpha_{1} \frac{N_{1}^{*}}{N_{2}^{*}}+\alpha_{2}+\alpha_{3} \frac{N_{3}^{*}}{N_{2}^{*}} .
$$

Hence $\zeta_{s}$, which is computational domain size in the transformed problem, corresponds to the total number of $U$ atoms in the truncated domain (per unit area), and this value is a constant.

The transformation leads to

$$
\frac{\partial}{\partial z} \rightarrow \varrho \frac{\partial}{\partial \zeta}, \quad \frac{\partial}{\partial t} \rightarrow \frac{\partial}{\partial t}-\varrho v \frac{\partial}{\partial \zeta},
$$


and the governing equations in the $(\zeta, t)$ framework, with $\epsilon=0$, are

$$
\begin{aligned}
& 0=\varrho \frac{\partial}{\partial \zeta}\left(D_{1,2} \varrho \frac{\partial \rho_{1,2}}{\partial \zeta}\right)+r_{1,2} \\
& \frac{\partial\left(\alpha_{1,2,3}\right)}{\partial t}+\varrho \frac{\partial v}{\partial \zeta} \alpha_{1,2,3}=R_{1,2,3} .
\end{aligned}
$$

The reaction rate densities $\left(r_{i}, R_{j}\right)$ are given by $(2.3)$ and $\varrho v_{\zeta}$ is given by the right-hand side of (2.6e).

To kickstart the reaction we need an initial quantity of either $\alpha_{1}$ or $\rho_{2}$, so we start from an initial condition that can be thought of as a slightly pre-oxidised state:

$$
\alpha_{2}(\zeta, 0)=\frac{1}{2}\left(1-\tanh \left(\zeta-\left(\zeta_{s}-\delta_{2}\right)\right)\right), \quad \alpha_{3}(\zeta, 0)=\frac{1}{2}\left(1+\tanh \left(\zeta-\left(\zeta_{s}-\delta_{3}\right)\right)\right),
$$

$$
\alpha_{1}(\zeta, 0)=1-\alpha_{2}(\zeta, 0)-\alpha_{3}(\zeta, 0),
$$

in the region $\zeta \in\left[0, \zeta_{s}\right]$ with $\delta_{2}=5$ and $\delta_{3}=4$. The precise choice of condition, provided that the reaction is started, does not affect the leading-order large-time behaviour. The numerical results are easily cast back into the $(z, t)$ framework once the solution has been determined.

\section{References.}

Baker, M., L. Less, and S. Orman (1966). Uranium + water reaction. Part 1. Kinetics, products and mechanism. T. Faraday Soc. 62, pp. 2513-2524.

Banos, A, N. Harker, and T. Scott (2018). A review of uranium corrosion by hydrogen and the formation of uranium hydride. Corros. Sci. 136, pp. 129-147.

Bazant, M. and H. Stone (2000). Asymptotics of reaction-diffusion fronts with one static and one diffusing reactant. Physica D 147.1-2, pp. 95-121.

Colmenares, C. (1975). The oxidation of thorium, uranium, and plutonium. Prog. Solid State Ch. 9, pp. 139-239.

Gálfi, L. and Z. Rácz (1988). Properties of the reaction front in an A $+\mathrm{B} \rightarrow \mathrm{C}$ type reaction-diffusion process. Phys. Rev. A 38.6, p. 3151.

Glascott, J. and I. Findlay (2019). Private communication.

Haycock, E. (1959). Transitions from parabolic to linear kinetics in scaling of metals. J. Electrochem. Soc. 106.9, pp. 771-775.

Hilhorst, D., R. Van Der Hout, and L. Peletier (1996). The fast reaction limit for a reaction-diffusion system. J. Math. Anal. Appl. 199.2, pp. 349-373.

Jiang, Z. and C. Ebner (1990). Simulation study of reaction fronts. Phys. Rev. A 42.12, p. 7483 .

Koza, Z. (1996). The long-time behavior of initially separated A $+\mathrm{B} \rightarrow 0$ reactiondiffusion systems with arbitrary diffusion constants. J. Stat. Phys. 85.1-2, pp. 179191.

- (1997). Asymptotic behaviour of initially separated A $+\mathrm{B}$ (static) $\rightarrow 0$ reactiondiffusion systems. Physica A: Stat. Mech. Appl. 240.3-4, pp. 622-634.

Léger, C., F. Argoul, and M. Z. Bazant (1999). Front dynamics during diffusion-limited corrosion of ramified electrodeposits. J. Phys. Chem. B 103.28, pp. 5841-5851.

Martin, T. et al. (2016). Atomic-scale studies of uranium oxidation and corrosion by water vapour. Sci. Rep. 6, p. 25618.

McGillivray, G., D. Geeson, and R. Greenwood (1994). Studies of the kinetics and mechanism of the oxidation of uranium by dry and moist air A model for determining the oxidation rate over a wide range of temperatures and water vapour pressures. J. Nucl. Mat. 208.1-2, pp. 81-97. 
Ostoja-Starzewski, M. and J. Schulte (1996). Bounding of effective thermal conductivities of multiscale materials by essential and natural boundary conditions. Phys. Rev. B 54.1, p. 278.

Peretz, M. et al. (1976). NMR study of hydrogen diffusion in uranium hydride. J. Phys. Chem. Sol. 37.1, pp. 105-111.

Polanowski, P. and Z. Koza (2006). Reaction-diffusion fronts in systems with concentration dependent diffusivities. Phys. Rev. E 74.3, p. 036103.

Ritchie, A. (1984). The kinetics and mechanism of the uranium-water vapour reaction - an evaluation of some published work. J. Nucl. Mat. 120.2-3, pp. 143-153.

Scott, T. et al. (2011). The oxidative corrosion of carbide inclusions at the surface of uranium metal during exposure to water vapour. J. Hazard Mater. 195, pp. 115123.

Winer, K. et al. (1987). Interaction of water vapor with clean and oxygen-covered uranium surfaces. Surf. Sci. 183.1-2, pp. 67-99. 